\title{
Quantum phase transition of fracton topological orders
}

\author{
Ting Fung Jeffrey Poon $\oplus^{1,2}$ and Xiong-Jun Liu $\odot^{1,2,3,4, *}$ \\ ${ }^{1}$ International Center for Quantum Materials, School of Physics, Peking University, Beijing 100871, China \\ ${ }^{2}$ Collaborative Innovation Center of Quantum Matter, Beijing 100871, China \\ ${ }^{3}$ CAS Center for Excellence in Topological Quantum Computation, University of Chinese Academy of Sciences, Beijing 100190, China \\ ${ }^{4}$ Institute for Quantum Science and Engineering and Department of Physics, Southern University of Science and Technology, \\ Shenzhen 518055, China
}

(Received 24 January 2020; accepted 2 November 2021; published 15 November 2021)

\begin{abstract}
Fracton topological order (FTO) is a new classification of correlated phases in three spatial dimensions with topological ground-state degeneracy (GSD) scaling up with system size and fractional excitations which are immobile or have restricted mobility. With the topological origin of GSD, FTO is immune to local perturbations, whereas a strong enough global external perturbation is expected to break the order. The critical point of the topological transition is either characterized by the broken GSD or the appearance of topologically distinct states with lower energy. In this work, we propose to characterize quantum phase transition of the type-I FTOs induced by external terms, when the transition can be characterized by the breaking down of GSD, and develop a theory to study analytically the critical points. In particular, for the external perturbation term creating lineon-type excitations, we predict a generic formula for the point of the quantum phase transition, characterized by the breaking down of GSD. This theory applies to a board class of FTOs, including X-cube model, and for more generic FTO models under perturbations creating two-dimensional (2D) or 3D excitations, we predict the upper and lower limits of the critical point. Our work makes a step in characterizing analytically the quantum phase transition of generic fracton orders.
\end{abstract}

DOI: 10.1103/PhysRevResearch.3.043114

\section{INTRODUCTION}

The discovery of topological quantum phases revolutionized the characterization and classification of fundamental states of quantum matter beyond the Landau symmetrybreaking paradigm. The extensive studies have brought about two broad categories of topological matter, the symmetryprotected topological phases [1-4] and the topologically ordered phases [5-7], which exhibit short- and long-range quantum entanglement, respectively. Unlike the topological phases which necessitate symmetry-protection, the topological orders are characterized by finite ground-state degeneracy (GSD), which is protected by the bulk gap and robust against any local perturbations, and host freely moving fractional excitations [8-11]. The topological protection of the GSD can have potential application to fault-tolerant quantum computation [12-14], particularly for the systems that host non-Abelian excitations [15-19].

Recently, a new basic class of topological phases, called fracton topological order (FTO) [20-28], was proposed and deeply broadened the understanding of the correlated topological states. In contrast to the conventional topological-ordered

\footnotetext{
${ }^{*}$ Corresponding author: xiongjunliu@pku.edu.cn

Published by the American Physical Society under the terms of the Creative Commons Attribution 4.0 International license. Further distribution of this work must maintain attribution to the author(s) and the published article's title, journal citation, and DOI.
}

phases, the GSD of FTO scales up with the system size, and the fractional excitations of a FTO are immobile or have restricted mobility [29-32]. In particular, two types of FTOs are proposed and distinguished by their excitations [24]. For type-I fracton orders, there are three categories of excitations, i.e., the one-dimensional (1D) lineon excitations, 2D and 3D fractons, which are generated by 1D line, 2D membrane, and $3 \mathrm{D}$ body operators, respectively. The fractons are located at the ends of the line operator or corners of the membrane and body operators and can move only along the line or in the way that the number of corners of the membrane/body operator remains the same. On the other hand, for the type-II fracton phases, the excitations are located at the corners of a fractal operator and are completely immobile [20]. The restricted mobility of excitations is important for the stability of FTOs at finite temperature [33,34] compared with conventional topological orders and may be advantageous in applying to topological quantum computation.

The exploration of the FTOs is still in the relatively early stage. Efforts have been made in relating the fracton orders to other novel physics [35-37], improving the basic concepts and characterizations of FTOs [38-40], and building new models for realization $[28,38]$. So far the studies are performed on the fine-tuned toy models, which are exactly solvable but hard to achieve in real physical systems. On the other hand, with the protection by the topological bulk gap, the FTOs are expected to be stable against small external perturbations and may undergo quantum phase transition when the system is tuned far away from the fine-tuning point. To uncover the critical point of such transition is crucial to determine the 
phase diagram, while very challenging in general, and may provide insights into the possible realization of FTOs in the real physical systems. This, therefore, brings a challenging but highly nontrivial issue to the frontier of this research direction, namely, characterization of the quantum phase transition of FTOs, as we study in this work.

In this article, we aim to characterize quantum phase transition in type-I FTOs induced by external perturbation terms. The GSD is unchanged when the strength of external term is less than a critical value. As the external term exceeds the critical value, the GSD either breaks down or is enhanced. For the former case (second-order transition), the GSD is lifted since there are nonzero transition matrix elements between some ground states at infinite system size. For the latter case (first-order transition), there are extra states, coming from excited states, reach the ground-state energy, and become new ground states as the external term is further increased. We develop an analytic theory for the former type of transitions. The universal results of the critical point of the GSD breaking down are obtained. The article is organized as follows. In Sec. II we briefly review the basics of FTOs. Then in Sec. III we define the quantum phase transition in FTOs and present the generic formalism. The analytic theory of the critical point of phase transition is presented in Sec. IV, with a universal result being obtained by mapping the present framework to a random-walk theory and applicable to a class of FTOs, including the $\mathrm{X}$-cube model. In Sec. V, we generalize the present study based on random-walk theory to more generic FTOs and show the upper limits of the critical points of the phase transition. A reasonable conjecture with numerical verification of the lower limit of the critical points is also presented. Finally, the conclusion is given in the last section.

\section{REVIEW OF FTO MODELS}

We start with a brief review of the basics of FTOs by introducing two typical models, the X-cube model [24] and the Haláz-Hsieh-Balents (HHB) model [25]. After that we shall introduce the outstanding issue on the quantum phase transition of FTOs, as studied in this work.

\section{A. X-Cube model}

The X-Cube model is a spin-1/2 model defined on the edges of a cubic lattice [24], with the Hamiltonian

$$
H_{\mathrm{X}-\mathrm{Cube}}=-\frac{w}{4}\left(\sum_{c} A_{c}+\sum_{v, i} B_{v}^{(i)}\right),
$$

where $c$ runs through all cube centered at $c$ and $A_{c}$ is a product of $12 \sigma_{x}$-operators defined on the edges of the cube, $v$ runs through all vertexes, and $B_{v}^{(i)}$ is a product of $4 \sigma_{z}$-operators defined on the edges which are attached to the vertex and are orthogonal to the direction $i . A_{c}$ and $B_{v}^{(i)}$ commute, so the ground state can be built from an eigenstate of all $B_{v}^{(i)}$. Moreover, since $A_{c}^{2}=B_{v}^{(i) 2}=1$, the ground states can be written in the following form,

$$
|g\rangle=\prod_{c} \frac{1+A_{c}}{\sqrt{2}}\left|\forall v, i, B_{v}^{(i)}=1\right\rangle .
$$

The forms of the ground states show clearly that the topological GSD originates from different choices of $\mid \forall v, i, B_{v}^{(i)}=$ $1\rangle$. The ground states thus generated can only be connected through flipping spins along straight lines that form noncontractible loops. In this model, the degeneracy is $2^{6 N-3}$ for the system with $N^{3}$ cubes.

There are two types of excitations [24]: (i) Lineonsgenerated by applying $\sigma^{x}$ on a ground state along an edge. The lineons are formed at the end points of the edge with energy $w / 2$. If $\sigma^{x}$ is applied on consecutive edges that form a straight line, two lineons will be formed on the head and on the tail. If the consecutive edges contain turning points, right at the turning points new excitations will be generated and cost extra energy. (ii) Fractons-generated by applying $\sigma^{z}$ on a ground state on the edges that form a membrane perpendicular to the direction of the edges. The fractons then form on the corners of the membrane, and the energy of each group of four fractons is $w$.

\section{B. HHB model}

The HHB model is a spin-1/2 model defined on a bodycentered cubic (bcc) lattice [25], where each lattice site contains four spins, and the Hamiltonian

$$
H_{\mathrm{HHB}}=-J \sum_{\mathbf{r} j} \sigma_{\mathbf{r}, j}^{x} \sigma_{\mathbf{r}+\mathbf{r}_{j}, j}^{y}-\lambda J \sum_{\mathbf{r}, j \neq j^{\prime}} \sigma_{\mathbf{r} j}^{z} \sigma_{\mathbf{r} j^{\prime}}^{z}
$$

Here $\mathbf{r}$ runs over different lattice sites, $j=A, B, C$, or $D$ for each flavor of spin and $\mathbf{r}_{A}, \mathbf{r}_{B}, \mathbf{r}_{C}$, and $\mathbf{r}_{D}$ are the shortest vectors connecting two sites in the direction $\{111\},\{\overline{1} 11\},\{1 \overline{1} 1\}$, and $\{11 \overline{1}\}$, respectively. If $\lambda \rightarrow \infty$, all spins in the same site are fully polarized. For $\lambda \gg 1$, the first term of the Hamiltonian can be treated as a perturbation, and the effective Hamiltonian at the leading order can be written as

$$
H_{\mathrm{HHB}, \mathrm{eff}}=-\frac{w}{32} \sum_{\mathbf{r}} \tilde{A}_{\mathbf{r}},
$$

where $\tilde{A}_{\mathbf{r}}=\prod_{s= \pm j} \tilde{\sigma}_{\mathbf{r}+s \mathbf{r}_{j}}^{y} \prod_{s= \pm, j=\{B, C, D\}} \tilde{\sigma}_{\mathbf{r}+s\left(\mathbf{r}_{A}-\mathbf{r}_{j}\right)}^{x}$ and $w \sim$ $J / \lambda^{31}$. The tilded Pauli matrices at $\mathbf{r}$ denote the corresponding Pauli matrices defined on the subspace of two ground states in each site $\mathbf{r}$ at $\lambda=\infty$. All $\tilde{A}_{\mathbf{r}}$ commutes and they have eigenvalues \pm 1 so that in the similarity of the deviation in the X-Cube model, the ground states can be written in the following form:

$$
|g\rangle=\prod_{e} \frac{1+\tilde{A}_{e}}{\sqrt{2}}\left|\forall o, \tilde{A}_{o}=1\right\rangle,
$$

where $e$ and $o$ are distinct subsets of the lattice sites such that each forms a simple cubic lattice that are translated in the $\{111\}$ direction.

Similarly, the topological GSD originates from different choices of $\left|\forall o, \tilde{A}_{o}=1\right\rangle$ and is $2^{12 N-10}$ for $2 N^{3}$ lattice sites. The system also hosts two different types of excitations. (i) Applying $\tilde{\sigma}_{x}$ on odd sites and $\tilde{\sigma}_{y}$ on even sites (or vice versa) along a straight line in the direction $\pm \mathbf{r}_{j}(j=A, B, C, D)$ yields a pair of lineon excitations. Unlike the X-Cube model, the straight lines can be packed together to form 2D membrane or $3 \mathrm{D}$ body operators. (ii) Applying $\tilde{\sigma}_{x}$ on the sites 
along a line in $\pm\left(\mathbf{r}_{A}-\mathbf{r}_{j \neq A}\right)$ directions also yields a pair of lineons. This operator can again be packed together to form 2D membrane or 3D body operators. Thus these types of excitations are classified as 3D fractons in our terminology.

\section{Overview and FTO breaking down}

All FTO models have topological GSD. The ground states can only be connected to each other through global operation, for example, the spin-flipping along noncontractible loops. Therefore, any local perturbations cannot break the GSD. However, a strong enough global perturbation can break the GSD. For example, we consider a transverse magnetic term on the X-cube model with

$$
H=H_{\mathrm{X}-\text { Cube }}+v \sum_{e} \sigma_{x, e},
$$

where $e$ runs through all edges of the cubic lattice. This external term can generate lineons on the end points of the edges. It is easy to see that for $v \rightarrow \infty$, there is only one ground state with all spins being aligned along the $-x$ direction, so a quantum phase transition must occur at some finite critical $v=v_{c}$, beyond which the FTO breaks down. This brings an outstanding question: How does the external term break the GSD? Answering the question is important to understand the topological phase region of FTO and is crucial for the realization of FTO in real physical systems without fine tuning. Currently the FTOs are given in toy models which can be solved analytically. With an addition of an external term, the system typically becomes not exactly solvable, and how to characterize the phase transition is a highly nontrivial but challenging issue.

\section{QUANTUM PHASE TRANSITION OF FTO: DEFINITION AND GENERIC FORMALISM}

\section{A. Define the quantum phase transition}

To approach this question, we consider the Hamiltonian in the following form:

$$
H=H_{0}+\bar{v} V(v),
$$

where $H_{0}$ is an unperturbed Hamiltonian of FTO with system size $N^{3}$, the external perturbation $V(v)$ is considered to the system with strength parameter $v$, and $\bar{v}$ is a dimensionless factor that will be taken as unity later in the study. The perturbation may induce couplings in the ground states and thus break the GSD. Let $\Delta E(N, v)$ be the energy splitting of the ground-state subspace. We shall see that,

$$
\begin{aligned}
& \lim _{N \rightarrow \infty} \Delta E\left(N, v<v_{c}\right)=0, \\
& \lim _{N \rightarrow \infty} \Delta E\left(N, v>v_{c}\right)>0 .
\end{aligned}
$$

This implies $v=v_{c}$ is a critical value, below which GSD is unchanged, while beyond which the GSD shall break down. In particular, we shall show that when $v<v_{c}$, for any two degenerate ground states $|g\rangle$ and $\left|g^{\prime}\right\rangle$, we have $\lim _{N \rightarrow \infty}\left\langle g\left|H_{\mathrm{eff}}\right| g^{\prime}\right\rangle=$ 0 , and for $v>v_{c}$, for any $|g\rangle$, there exists other ground states $\left|g^{\prime}\right\rangle$ and $\lim _{N \rightarrow \infty}\left\langle g\left|H_{\text {eff }}\left(v>v_{c}\right)\right| g^{\prime}\right\rangle \rightarrow \infty$. Here $H_{\text {eff }}$ is an effective Hamiltonian defined on the subspace of the ground states that capture the transitions among them. Note that to (a)

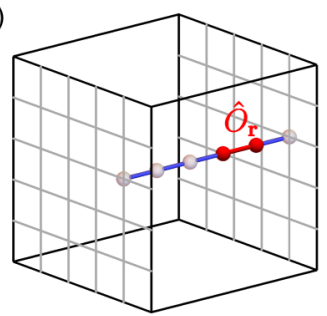

(b)

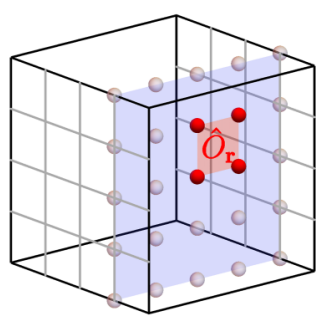

(c)

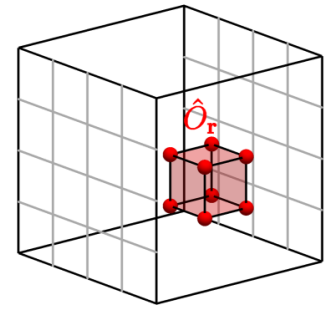

FIG. 1. Schematic diagrams for the definition of $\hat{O}_{\mathbf{r}}$ when it produces (a) lineons, (b) 2D fractons, or (c) 3D fractons in the vicinity. The red circles denote the lineons (2D fractons) produced by the operation of $\hat{O}_{\mathbf{r}}$. The operation of $\hat{O}$ can also annihilate the excitations for those points which excitation is already present.

avoid the finite-size effect, the system size $N$ must be taken as infinity.

\section{B. A general framework}

We consider that the external extra term is written in the following generic form of fracton operators:

$$
V=v \sum_{\mathbf{r}} \hat{O}_{\mathbf{r}}
$$

where $\hat{O}_{\text {r }}$ generates or annihilates two lineons [Fig. 1(a)], four 2D fractons [Fig. 1(b)], or eight 3D fractons [Fig. 1(c)] at its vicinity. In Fig. 1, the operator $\hat{O}_{\mathbf{r}}$ is denoted by a red line, square, or cube, respectively, and the excitations created by it are represented by the red spheres.

To facilitate the study on how the external term $V(v)$ changes GSD, we split the Hilbert space into two subspaces,

$$
\psi=\left(\begin{array}{c}
\psi_{g} \\
\psi_{\bar{g}}
\end{array}\right),
$$

where $\psi$ represents the full Hilbert space, $\psi_{g}$ denotes the ground-state subspace, and $\psi_{\bar{g}}$ denotes the subspace of the states with excitations. With this notation, we project the whole Hamiltonian onto the ground-state subspace and obtain the effective Hamiltonian (Appendix),

$$
\begin{aligned}
H_{\mathrm{eff}}= & H_{0, g g}+\bar{v} V_{g g}-\sum_{i=2}^{\infty} \bar{v}^{i} V_{g \bar{g}} \\
& \times\left(\sum_{\left(s_{1}, \cdots, s_{m}\right) \in P_{i-1}} Q^{\left(s_{1}\right)} V_{\bar{g} \bar{g}} \cdots V_{\bar{g} \bar{g}} Q^{\left(s_{m}\right)}\right) V_{\bar{g} g} .
\end{aligned}
$$

$P_{i-1}$ is the set of integer partitions of $i-1$, i.e., $s_{1}+s_{2}+$ $\cdots+s_{m}=i-1$. The subscripts $g g$ (or $\bar{g} \bar{g}$ ) means that the corresponding operator is projected onto the ground-state subspace (or the excited state subspace). Similarly, the subscripts $g \bar{g}$ and $\bar{g} g$ mean that the corresponding operators connect the 
ground state with the excited state subspace. Finally, $Q^{(s)}$ 's are the factors of inverse energy scale, which denotes the energy difference between the ground state and intermediate excited states of the FTO (see more details in Appendix). Since only the operation on noncontractible loops can induce transition between two different ground states, the leading-order correction to the ground-state subspace must be the $N$-th order. In the leading-order contribution, only the terms with $Q^{(1)}=$ $\left(E_{0}-H_{0, \bar{g} \bar{g}}\right)^{-1}$ have a nonzero matrix element between two different ground states. Therefore, the effective Hamiltonian by taking into account the leading-order contribution reads

$$
H_{\mathrm{eff}}^{(N)}=-\bar{v}^{N} V_{\bar{g} \bar{g}}\left(Q^{(1)} V_{\bar{g} \bar{g}} \cdots V_{\bar{g} \bar{g}} Q^{(1)}\right) V_{\bar{g} g} .
$$

Any lower-order perturbation cannot couple different ground states, since a noncontractible loop must be formed by at least $N$ times of $\hat{O}_{\mathbf{r}}$ operations.

The energy splitting in the ground states depends on two aspects. The first is the coupling between each two ground states (e.g., $|g\rangle$ and $\left|g^{\prime}\right\rangle$ ) up to all higher orders. In particular, the matrix element for the $(N+r)$ th-order contribution is given by $\mathcal{H}_{\mathrm{eff}, g g^{\prime}}^{(N+r)}=\left\langle g\left|H_{\mathrm{eff}}^{(N+r)}\right| g^{\prime}\right\rangle$, with $r=0$ being the leading-order contribution. The second is the number of ground states $\left|g^{\prime}\right\rangle$ which are coupled to a certain fixed ground state $|g\rangle$ by the external term, denoted by $f^{(N+r)}$. The energy splitting from the asymptotic behavior should be given by

$$
\Delta E \sim \sum_{r=0}^{\infty} f^{(N+r)} \mathcal{H}_{\mathrm{eff}, g g^{\prime}}^{(N+r)}, N \rightarrow \infty .
$$

Intuitively, the ground-state subspace can be treated as a flat band of strongly correlated many-body states before phase transition. For the strength of external perturbation exceeding a critical value, the flat band is deformed to be a dispersive one, with $\Delta E$ being the bandwidth.

\section{CRITICAL POINT OF THE QUANTUM PHASE TRANSITION}

We proceed to study the key issue, the critical point of the quantum phase transition in the FTOs. We shall show that, for the external perturbation creating only lineon-type excitations, the critical point of the phase transition can be given by a universal formula and in some special cases the result given by the formula is exact, as presented in the following theorem.

Theorem 1. Let $w$ be the energy of a pair of separated lineons and $w_{i}$ be the energy of two lineons along different directions crossing at an intersecting site. If the system does not undergo a first-order phase transition, then the quantum phase transition occurs exactly at $v=v_{c 1}=\frac{w}{4}$ if $w_{i} \geqslant w$ or $v=v_{c 1}^{\prime} \approx \frac{w}{4} \times\left(1-\frac{w-w_{i}}{w} \times 0.26\right)$ if $w_{i}<w$. This theorem can be proved by finding a critical $v$ such that the leading-order and all higher-order contributions of the transition matrix element between different ground states in the thermodynamical limit is nonzero. We first begin our discussion on the general properties of the contributions of different order in the subsections A and B. In the latter part of subsection B and subsection $\mathrm{C}$, we assume that $f=w$ and prove the theorem in this regime. In subsection $\mathrm{D}$, we argue that the exact result can extend to the case $f>w$ and employ a similar method to give a universal formula for $f \leqslant w$ under certain approximation.

\section{A. The leading-order contribution}

We start with the leading-order contribution to the matrix element of $H_{\text {eff }}$ between two ground states $|g\rangle$ and $\left|g^{\prime}\right\rangle$. Let the two ground states be connected by a noncontractible straight line of $\hat{O}_{\text {r }}$ operators, i.e.,

$$
\left|g^{\prime}\right\rangle=\prod_{r} \hat{O}_{\mathbf{r}}|g\rangle
$$

where $\mathbf{r}$ runs through all sites along a straight line. Denote by $V_{\gamma \gamma^{\prime}}=\left\langle\gamma|V| \gamma^{\prime}\right\rangle$ the matrix element of $V(v)$ and $F_{\gamma}$ the number of pairs of generated lineons at the intermediate states $|\gamma\rangle$. The matrix element of the effective Hamiltonian reads

$$
\begin{aligned}
\mathcal{H}_{\mathrm{eff}, g g^{\prime}}^{(N)} & =\left(\frac{-1}{w}\right)^{N-1} \sum_{\left\{\beta_{1}, \cdots, \beta_{N-1}\right\}} \frac{V_{g \beta_{1}} V_{\beta_{1} \beta_{2}} \cdots V_{\beta_{N-1} g^{\prime}}}{F_{\beta_{1}} F_{\beta_{2}} \cdots F_{\beta_{N-1}}}, \\
& =v\left(\frac{-v}{w}\right)^{N-1} \sum_{\left\{\beta_{1}, \cdots, \beta_{N-1}\right\}} \frac{1}{F_{\beta_{1}} F_{\beta_{2}} \cdots F_{\beta_{N-1}}} .
\end{aligned}
$$

The denominator implies that the energy of the intermediate state depends purely on the number of lineons in the system. The summation is over all permutation of $\left\{\beta_{1}, \cdots, \beta_{N-1}\right\}$; the last line in the above equation is obtained by noticing that $V_{\gamma \gamma^{\prime}}$ is either 0 or $v$, and the prime means that the summation is over all the intermediate states with $V_{\gamma \gamma^{\prime}}=v$. As an example, when $N=6$, one of the terms in the summation is shown in Figs. 2(a) and 2(b), where the number of lineons $F_{\beta_{1 \sim 5}}$ of the intermediates states $\left|\beta_{1 \sim 5}\right\rangle$ are 1, 2, 2, 1, and 1, respectively.

Exactly counting the summation in Eq. (15) is highly nontrivial when $N$ is large. With the details presented in the supplemental material [41], we find that the exact value of the leading-order perturbation takes a novel form

$$
\mathcal{H}_{\mathrm{eff}, g g^{\prime}}^{(N)}=v\left(-\frac{v}{w}\right)^{N-1} C_{2 N-2}^{N-1} \simeq \frac{v}{\sqrt{\pi N}}\left(-\frac{4 v}{w}\right)^{N-1} .
$$

The leading-order contribution is deeply related to the socalled Catalan number [41], $C_{2 N-2}^{N-1} / N$, as illustrated in Fig. 2(c). The $N$ th Catalan number is defined as the number of paths along the edges of a grid with $N \times N$ square cells from the bottom-left corner (purple dot) to the top-right corner (green dot) with two restrictions. (i) Each step of the path has to be either rightward or upward. (ii) The whole path has to be below the diagonal line (painted red). Or equivalently, the path has to pass through $N$ odd lattices below the diagonal, including the lattice rightward to the bottom-left corner $\left(b_{i}\right)$, the lattice downward to the top-right corner $\left(b_{f}\right)$, and $N-2$ more odd lattices (circles on the vertexes of the grid). In particular, the $N-1$ th Catalan's number $\mathcal{C}_{N-1}$ is related to the leading-order perturbation by $\mathcal{H}_{\mathrm{eff}, \mathrm{gg}^{\prime}}^{(N)} \propto N \mathcal{C}_{N-1}$.

To better understand the leading-order contribution, we show below that it can be mapped to a random-walk problem, which has the important merit to facilitate the further study of higher-order contributions and the more generic extra terms creating 2D/3D fractons. More specifically, we consider a random walk starting at $L_{1,1}$ in a network $L_{i, j}$, where $1 \leqslant$ $i \leqslant \min (j, N-j)$ equals the number of pairs of lineons in the $j$-th step of the intermediate process, with $1 \leqslant j \leqslant N-1$, 
(a)

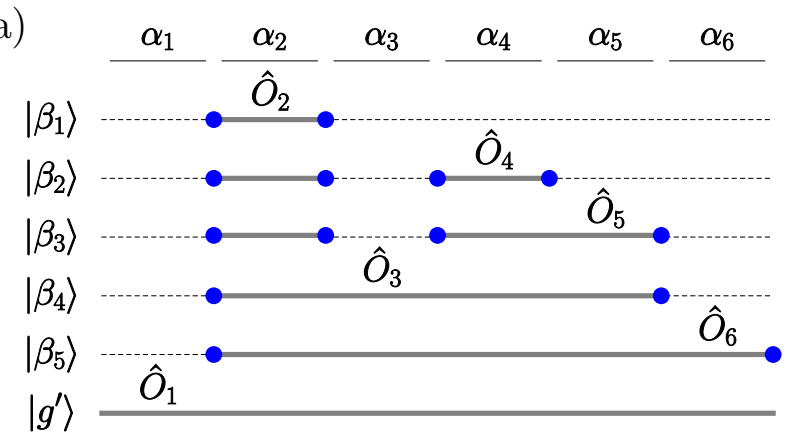

(b)

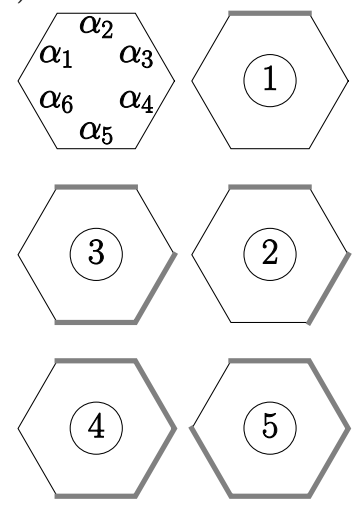

(c)

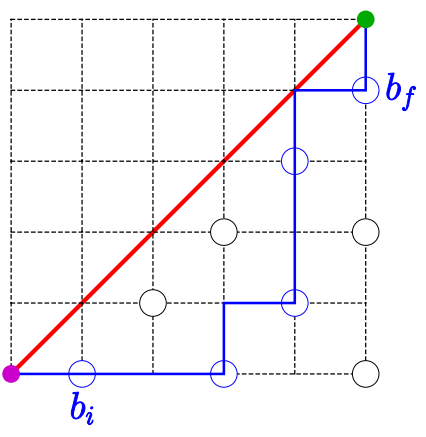

FIG. 2. (a), (b) One possible transition described in $H_{\text {eff }}^{(N=6)}$ from $|g\rangle$ to $\left|g^{\prime}\right\rangle . \hat{O}_{2}, \hat{O}_{4}, \hat{O}_{5}, \hat{O}_{3}, \hat{O}_{6}$, and $\hat{O}_{1}$ are applied on the groundstate $|g\rangle$ successively to produce $\left|\beta_{1 \sim 5}\right\rangle$ and $\left|g^{\prime}\right\rangle ; \alpha_{i}$ are lattices that

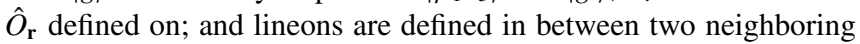
lattices and are shown as blue dots. (c) Schematic diagram showing the definition of 5th Catalan's number $\mathrm{C}_{5}$.

and moving $N-2$ steps with probabilities in each step [41],

$$
\begin{aligned}
\left.P\right|_{L_{i, j} \rightarrow L_{i-1, j+1}} & =\frac{i(i-1)}{(N-j)(N-j-1)}, \\
\left.P\right|_{L_{i, j} \rightarrow L_{i, j+1}} & =\frac{2 i(N-i-j)}{(N-j)(N-j-1)}, \\
\left.P\right|_{L_{i, j} \rightarrow L_{i+1, j+1}} & =\frac{(N-i-j)(N-i-j-1)}{(N-j)(N-j-1)} .
\end{aligned}
$$

For each step, we set the reward gained by the random walk as $1 / i$ so that the mean of the product of rewards matches the matrix element $\mathcal{H}_{\text {eff, } g g^{\prime}}^{(N)}$ after summing over all paths, except for the prefactor $v(-v / w)^{N-1}$. The exact result is given by

$$
\mathcal{H}_{\mathrm{eff}, g g^{\prime}}^{(N)}=\sum_{i_{2}=1}^{2} \sum_{i_{3}=1}^{3} \cdots \sum_{i_{N-2}=1}^{2} \frac{\left.\prod_{j=1}^{N-2} P\right|_{L_{i_{j}, j} \rightarrow L_{i_{j+1}, j+1}}}{\prod_{j=1}^{N-1} i_{j}},
$$

with $i_{1}=i_{N-1}=1$. A simple example with $N=6$ of the random-walk network is shown in Fig. 3. The shaded path shows a case where $i_{2}=i_{3}=2$ and $i_{4}=1$, and it corresponds to the sum of all possible intermediate states so that $F_{\beta_{1 \sim 5}}$ are $1,2,2,1$, and 1 , respectively. One example of such states is shown in Fig. 2(a). The sum of the rewards of the random walk equals the $N$ times of the Catalan number, recovering the result in Eq. (16).

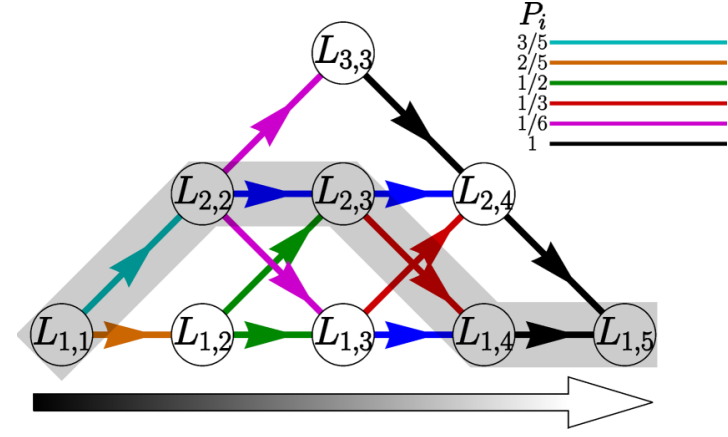

FIG. 3. An equivalent random-walk network for $N=6$. The shaded path depicts the sum of all possible intermediate states so that $F_{\beta_{1 \sim 5}}$ are 1,2, 2, 1, and 1, respectively, which Figs. 2(a) and 2(b) depict one example of such paths.

\section{B. Higher-order contributions}

The mapping to a random-walk problem can be generalized to the study of all higher-order contributions $\mathcal{H}_{\text {eff, } g g^{\prime}}^{(N+r)}$, with $r$ being even integers. Note that the odd $r$ is forbidden since the effective Hamiltonian with an addition of an odd number of $\hat{O}_{\mathbf{r}}$ operations must scatter ground states to excited ones. It is convenient to consider the higher-order terms in the two different cases, namely, the higher-order contribution with finite $r$ and the contribution with infinite $r$, respectively.

To get the intuition of the higher-order contributions, we consider first the lowest higher-order term with $r=2$. This type of higher-order terms contains $N+2$ number of $\hat{O}_{\mathbf{r}}$ operators and some of them have to be repeated. Since $\hat{O}_{\mathbf{r}}^{2}=1$, the repetition is actually equivalent to a backward step in the random-walk process. For $r=2$, the network $L$ is allowed to move back by one step at an intermediate step. As a simple example with $N=6$ and $r=2$ shown in Fig. 4(a), the $\hat{O}_{4}$ operation is repeated at the third step. When represented by the random walk, this process is equivalent to moving back at the second step. Specifically, the network $L$ is expanded to a new one $L^{(1)}$, in which the random walk includes a new boxed subnetwork to account for the process of moving backward by two steps, as shown in Fig. 4(b). For the generic configuration, let $P_{i}$ be the probability that the walker moves back at $i$-th step. The average reward, and so the matrix element, can be approximated as

$$
\mathcal{H}_{\mathrm{eff}, g g^{\prime}}^{(N+2)} \approx \sum_{i=3}^{N} P_{i} R_{N, i}
$$

Here $R_{N, i}$ is the average reward of the random-walk process which includes the backward moving at the $i$-th step. It can be shown that whenever $P_{i}$ is finite, the asymptotic behavior of $R_{N, i}$ remains the same as $\mathcal{H}_{\text {eff }, g g^{\prime}}^{(N)}$ for large $N$ (see supplemental material [41]). This further determines the asymptotic behavior of $\mathcal{H}_{\text {eff, } g g^{\prime}}^{(N+2)}$. For generic finite $r$, the above argument iterates $r / 2$ times to form a new network $L^{(r / 2)}$ and the similar result can be obtained. It then follows that the matrix element $\mathcal{H}_{\text {eff }, g g^{\prime}}^{(N+r)}$ with finite $r$ should be dominated by $(4 v / w)^{N+r}$ and the sum of the perturbation series up to such higher orders is exponentially small if $v<w / 4$. 

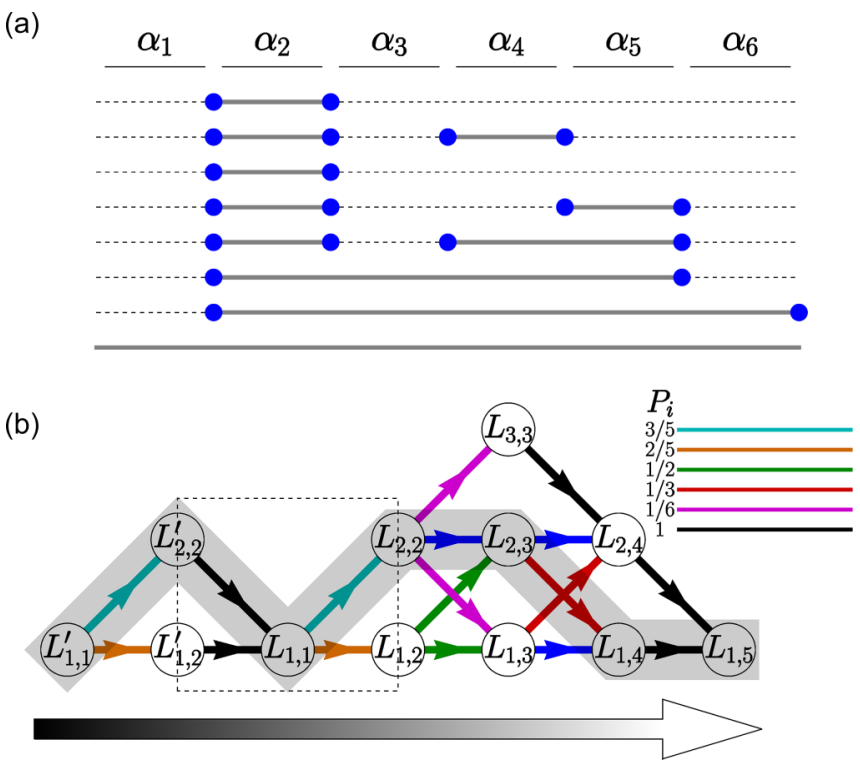

FIG. 4. (a) One possible way to transit from $|g\rangle$ to $\left|g^{\prime}\right\rangle$ described by $H_{\mathrm{eff}}^{(N+r)}$, where $N=6$ and $r=2$. (b) A transformed network $L^{(1)}$ so that the random walker moves back at the second step. The shaded path depicts the sum of all possible intermediate states so that $F_{\beta_{1 \sim 7}}$ are $1,2,1,2,2,1$, and 1 , respectively, which (a) depict one example of such paths. The dashed box indicates the inserted part of the new network $L^{(1)}$ to account for the step that moves backward in the original network $L$.

The remaining question is how the perturbation grows as $r$ tends to infinity, noting that the iteration cannot be done by an infinite number of times. For this purpose, we first assume that the energy of two lineons coming from mutually different directions meeting at the same point is $2 \times w / 2=w$, i.e., lineons coming from different directions are independent. Let $r=\zeta N$, where $\zeta$ is a real number. After some algebra we find for higher-order perturbations with infinite $r$ that [41]

$$
\lim _{N \rightarrow \infty} \frac{\mathcal{H}_{\mathrm{eff}, g g^{\prime}}^{(N+\zeta N+1)}}{\mathcal{H}_{\mathrm{eff}, g g^{\prime}}^{(N+\zeta N)}}=\frac{\bar{\lambda}}{N} \times \frac{v}{w},
$$

where $\bar{\lambda}=N / 4$ is the reward average over all possible configurations, i.e., all possible intermediate states connecting $|g\rangle$ with $\left|g^{\prime}\right\rangle$. The above is an important novel result and can be understood in the following way. For the higher-order term with infinite $r$, the network $L^{(r / 2)}$ allows to move backward an infinite number of times so that the process is close to an unrestrained random walk, namely, the random walk can perform in arbitrary directions. Thus the growth of the average of product of rewards is captured by the reward averaging over all possible configurations. With this one can show that the matrix element grows by $4 v / w$ as $r$ increases one order [41].

\section{Energy splitting}

Summing over all-order contributions yields the total transition matrix element

$$
\mathcal{H}_{\mathrm{eff}, g g^{\prime}}=\sum_{r} \mathcal{H}_{\mathrm{eff}, g g^{\prime}}^{(N+r)}=\sum_{r} \mathfrak{C}^{(r)}\left(\frac{4 v}{w}\right)^{(N+r)} .
$$

(a)

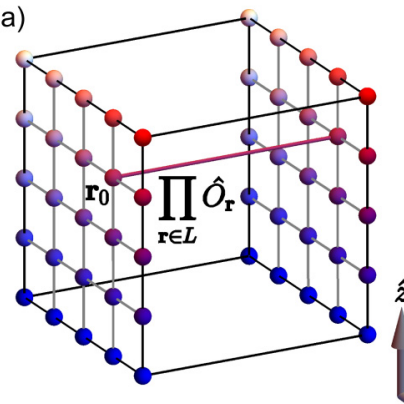

(b)

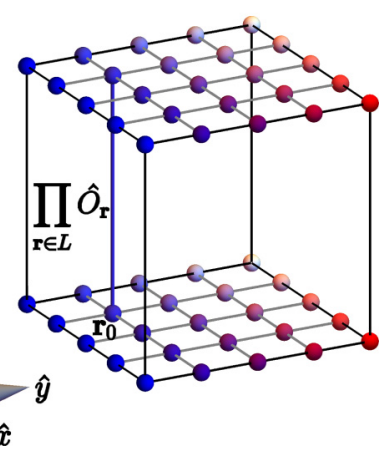

FIG. 5. Schematic diagrams showing the order of magnitude of $f^{(N)}$. The lines in the diagrams represent the product of operators $\hat{O}_{\text {r }}$ along a straight line $L$ to transform a ground state to another. Each choice of $\mathbf{r}_{0}$ represents one possible transformation and so each choice contributes 1 to $f^{(N)}$ so that pairs of surfaces give contributions of $N^{2}$ to $f^{(N)}$. Panels (a) and (b) show two examples that the pairs of surfaces are oriented in the $\hat{y}$ and $\hat{z}$ directions, respectively. This means that the final $f^{(N)}$ is of the order of $N^{2}$.

As the growth of $\mathcal{H}_{\mathrm{eff}, g g^{\prime}}^{(N+r)}$ is $4 v / w$ as $r \rightarrow \infty$, the growth of prefactor $\mathfrak{C}^{(r)}$ is subexponential. On the other hand, the number $f^{(N)}$ obeys power law of the system size and is of the $\operatorname{order} N^{2}$. This is because for a reference ground-state $|g\rangle$, the number of other ground states that can be coupled to it through

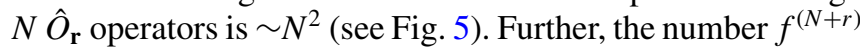
does not grow until $r$ is comparable to $N$, since an addition of a finite number of $\hat{O}_{\text {r }}$ operations couples no new ground states. Thus the number $f^{(N+r)}$ also grows subexponentially versus $N$ and $r$. With these results we conclude from Eq. (13) that the system, which we assume the lineons coming from different directions are independent, has a critical point given by

$$
v_{c 1}=\frac{w}{4} \text {. }
$$

For the perturbative term weaker than the above value, the GSD of the FTO is unaffected.

We provide an intuitive understanding of the above critical point by partially relating the perturbative study to the $1 \mathrm{D}$ transverse Ising model. Consider the case for two ground states $|g\rangle$ and $\left|g^{\prime}\right\rangle$ which can be connected through applying $\hat{O}_{\mathbf{r}}$ operators on a single straight line, i.e., $|g\rangle=\prod_{r \in L} \hat{O}_{\mathbf{r}}\left|g^{\prime}\right\rangle$. Here $L$ is a straight line forming a noncontractible loop. We further consider the following Hamiltonian $\tilde{H}=\tilde{H}_{0}+\tilde{H}_{1}$ :

$$
\tilde{H}_{0}=\frac{w}{2} \sum_{i} \hat{\xi}_{i, i+1}, \quad \tilde{H}_{1}=v \sum_{i} \hat{O}_{i},
$$

where $\hat{\xi}_{i, i+1}$ is an operator with eigenvalue 1 when there is a lineon between site $i$ and $i+1$ and 0 when there is not. $\hat{O}_{i}$ remove (create) when there is (not) a lineon in the vicinity of the site $i$. It then follows that

$$
\left[\hat{O}_{i}, \xi_{j, j+1}\right]=\hat{O}_{i}\left(1-2 \hat{\xi}_{j, j+1}\right)\left(\delta_{i j}+\delta_{i, j+1}\right) .
$$

With the above results we can verify that the matrix element of $\tilde{H}$ between $|g\rangle$ and $\left|g^{\prime}\right\rangle$ is identical to $\mathcal{H}_{\text {eff, } g g^{\prime}}$. Consider now the mapping that $\hat{\xi}_{i, i+1} \rightarrow\left(1+\sigma_{i}^{z} \sigma_{i+1}^{z}\right) / 2$ and $\hat{O}_{i} \rightarrow \sigma_{i}^{x}$, which keeps the above commutation relation (23). This leads to $\tilde{H} \rightarrow H_{\text {Ising }}=\frac{w}{4} \sum_{i} \sigma_{i}^{z} \sigma_{i+1}^{z}+v \sum_{i} \sigma_{i}^{x}$, which is the $1 \mathrm{D}$ 
transverse Ising model with the critical point given by $v_{c}=$ $w / 4$. From this result we can see that at the critical point the bulk gap of the FTO transition should close. It is noteworthy that this mapping is valid for part of the configurations, not exactly identical to the total 3D effective Hamiltonian (11), but gives the correct critical point if the lineons coming from different directions are independent. Also the mapping is valid only for the regime before the GSD is lifted.

\section{Correction due to intersecting lineons}

More generally, the result of critical value for system with lineon excitation depends on the energy $w_{i}$ of two lineons along different directions crossing at the intersecting site. (i) $w_{i}=w$. The global line operators can be treated as independent so the critical point is $v=v_{c 1}=w / 4$. (ii) $w_{i}>w$. Then the matrix elements between two arbitrary ground states may be finite only at some value $v>w / 4$. However, the critical point remains at $v=v_{c 1}=w / 4$ since the leadingorder contribution between $|g\rangle$ and $\left|g^{\prime}\right\rangle$ that are connected by a single global line operator is finite at $v=v_{c 1}=w / 4$. (iii) $w_{i}<w$. The above calculated critical point is overestimated since the energies of the intermediate steps are overestimated. It can be seen from Eq. (15) or the corresponding formula for higher-order contributions that matrix elements of the effective Hamiltonian are proportional to the inverse of the product of energies of intermediate states. Therefore, the critical point depends on the average correction to the intermediate energies. To capture the effect analytically, a factor $\mathfrak{f}^{(r)}$ is inserted into the expression (20) so that $\mathcal{H}^{\prime}$ eff, $g g^{\prime}=$ $\sum_{r} \mathfrak{C}^{(r)}\left(4 v \mathfrak{f}^{(r)} / w\right)^{N+r}$. An estimation of the correction factor can be given below (details are found in the supplemental material [41]).

We start the estimation by noting that the correction factor can be written as

$$
\left(\mathfrak{f}^{(r)}\right)^{N+r}=\left\langle\prod_{i} \mathfrak{f}_{\beta, i}^{(r)}\right\rangle_{\beta}=\frac{\sum_{\beta} \prod_{i}^{N+r} F_{\beta_{i}}^{-1} \mathfrak{f}_{\beta, i}^{(r)}}{\sum_{\beta} \prod_{i}^{N+r} F_{\beta_{i}}^{-1}},
$$

where $i$ denotes the $i$-ith intermediate step, $\langle\cdots\rangle_{\beta}$ denotes the weighted average over all configurations $\beta$, and $\mathfrak{f}_{\beta, i}$ captures the ratio of energy changes due to intersecting lineons. For example, if there are four nonintersecting lineons and two intersecting in a given configuration and the energy of each intersecting lineon is $w / 3$, then $\mathfrak{f}_{\beta_{i}}=(4 \times w / 2+$ $2 \times w / 3) /(6 \times w / 2)=9 / 8$. We start the estimation of $\mathfrak{f}^{(r)}$ by assuming $\mathfrak{f}_{\beta, i}$ to depend on $F_{\beta_{i}}$ only. This assumption is reasonable since the probability of lineons to be intersecting is proportional to the density of lineons. With this assumption, the correction factor becomes

$$
\left(\mathfrak{f}^{(r)}\right)^{N+r} \approx \frac{\sum_{F_{\beta_{1}} F_{\beta_{2}} \ldots} \prod_{i}^{N+r} M_{\beta_{i}} F_{\beta_{i}}^{-1} \mathfrak{f}_{F_{\beta_{i}}}^{(r)},}{\sum_{F_{\beta_{1}} F_{\beta_{2}} \ldots \prod_{i}^{N+r} M_{\beta_{i}} F_{\beta_{i}}^{-1}}}
$$

where $M_{\beta_{i}}$ is the number of configurations for a given $F_{\beta_{i}}$. The correction factor $\mathfrak{f}_{F_{\beta_{i}}}^{(r)}$ can be calculated for each $F_{\beta_{i}}$ and the value of $\mathfrak{f}_{F_{\beta_{i}}}^{(r)}$ where $M_{\beta_{i}} F_{\beta_{i}}^{-1}$ maximizes is [41],

$$
\mathfrak{f}_{\mathrm{dom}}^{-1} \approx 1-\frac{w-w_{i}}{w} \times 0.26
$$

where $w_{i} \leqslant w$ is the energy of two intersecting lineons and the critical point of this more generic case can be approximated by

$$
v_{c 1}^{\prime}=v_{c 1} \times \mathfrak{f}_{\text {dom }}^{-1} .
$$

This result is universal for the type of extra terms that create only lineon excitations. In particular, the quantum phase transition of the transverse field X-cube model, as given in Eq. (6), can directly obtained from the above result, and $v_{\mathrm{X} \text {-Cube }} \approx$ $(0.87 \pm 0.04) \times w / 4$. The origins of the error are discussed in the supplemental material [41]. This analytic result is close to the numerical result $v_{\mathrm{X} \text {-Cube }}^{\mathrm{pCUT}} \approx 0.9196 \times w / 4$ by the method of perturbative continuous unitary transformations [42].

\section{CRITICAL POINTS FOR THE GENERIC EXTRA TERMS}

The random-walk theory developed based on the effective Hamiltonian approach can be further applied to study the more generic cases beyond lineon-type perturbations. For those cases, the external perturbation can induce also the 2D and/or 3D fractons, e.g., for transverse field coupling to $\tilde{\sigma}_{x, y}$ in the HHB model, namely, the term $\hat{O}_{\mathbf{r}}$ generates or annihilates four 2D fractons [as shown in Fig. 1(b)] or eight 3D fractons [as shown in Fig. 1(c)] nearby the site r. We expect that when the strength $v$ of the extra term $V(v)$ exceeds certain critical value, the phase transition occurs and the FTO breaks down. Similar to the study for lineon-type perturbations, the exact critical points can be obtained in three key steps. (i) Identify how the leading-order contribution grows as $N \rightarrow \infty$. This encodes also how the higher-order contributions grow with finite $r$. (ii) With fixed $N$, identify how the infinite- $r$ higher-order contributions grow. (iii) Summing over all order contributions to obtain the energy splitting. Similarly, for a fixed ground-state $|g\rangle$, the number $f^{(N+r)}$ of $\left|g^{\prime}\right\rangle$ coupled to it through leadingorder perturbation must be power-law function in $N$. This value does not grow until $r$ is comparable to or over $N$ (for $2 \mathrm{D}$ and 3D fractons). Thus for $N \rightarrow \infty$ the growth of $f^{(N+r)}$ is again subexponential.

\section{A. Upper limits}

The exact result of the critical point is not analytically available in the current stage. The challenge arises from working out the leading-order contribution, as studied later. However, the higher-order contributions with infinite $r$ can be exactly obtained by the random-walk theory. For the case with 2D or 3D fractons, the sole consideration of (ii) gives an upper limit $v_{c}^{(u)}$ of the critical point, as stated below.

Theorem 2. For extra terms creating 2D fractons through membrane of $\hat{O}_{\mathbf{r}}$ operators in the FTO, the critical point $v_{c 2}$ of the phase transition satisfies $v_{c 2} \leqslant v_{c 2}^{(u)}=3 w / 16$. For extra terms creating 3D fractons through body operators, the critical point $v_{c 3}$ satisfies $v_{c 3} \leqslant v_{c 3}^{(u)}=19 w / 256$. Here $w$ is the energy of a group of four (or eight) fractons of the 2D membrane (3D cube). Since the broken GSD implies that quantum phase transition must occur, the above upper limit of the critical point is true for any type of quantum phase transitions.

In particular, we show for the $2 \mathrm{D}$ fracton case the growth of $\mathcal{H}_{\mathrm{eff}, \mathrm{gg}^{\prime}}^{(N+r)}$ is $16 v / 3 w$ as $r=\zeta N$ increases by one order when $|g\rangle$ and $\left|g^{\prime}\right\rangle$ is connected by a 2D membrane of operators $\hat{O}_{\mathbf{r}}$. This can be seen by noticing that at any point $\mathbf{r}_{0}$ (on which a 
(a)

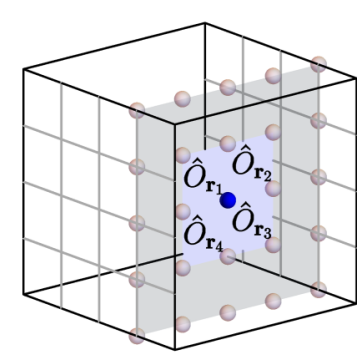

(b)

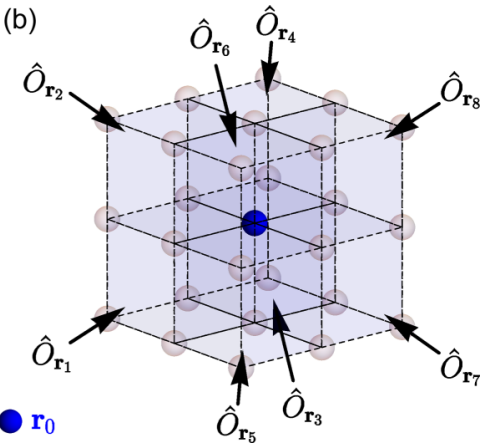

$r_{0}$

FIG. 6. Schematic diagrams showing the definition of the operators $\hat{O}_{\mathbf{r}_{i}}$ surrounding the point $\mathbf{r}_{0}$ (blue sphere) on which a (a) $2 \mathrm{D}$ or (b) $3 \mathrm{D}$ fracton is defined. The (a) membrane or (b) body operators $\hat{O}_{\mathbf{r}_{i}}$ are printed blue.

2D fracton is defined) that are surrounded by four operators $\hat{O}_{\mathbf{r}_{1}}, \hat{O}_{\mathbf{r}_{2}}, \hat{O}_{\mathbf{r}_{3}}$, and $\hat{O}_{\mathbf{r}_{4}}$ [listed in clockwise direction, as shown in Fig. 6(a)], the number of fractons $F_{2, \mathbf{r}_{0}}$ at $\mathbf{r}_{0}$ can be written as

$$
F_{2, \mathbf{r}_{0}}=\sum_{i=1}^{4}\left(s_{i}-2 s_{i} s_{i+1}+2 \prod_{j \neq i} s_{j}\right)-4 \prod_{i} s_{i},
$$

where $s_{5}=s_{1}$ and $s_{i}=\left(1+\hat{O}_{r_{i}}\right) / 2$. As shown through random-walk theory in Sec. IV B, the growth of high-order contributions with infinite $r$ is proportional to the average number $\bar{F}_{2, \mathbf{r}_{0}}$ of excitations over all possible configurations. Note that each term of $s_{i}$ contributes a factor of $1 / 2$. It then follows that $\bar{F}_{2, \mathbf{r}_{0}}=3 / 4$. Therefore, the growth required is $4 \times 4 v / 3 w=16 v / 3 w$, giving the upper limit of the critical value $v_{c 2} \leqslant v_{c 2}^{(u)}=3 w / 16$.

The upper limit of the critical point can be obtained for the case with $3 \mathrm{D}$ fractons in a similar way. At any point $\mathbf{r}_{0}$ that is surrounded by eight $\hat{O}_{\mathbf{r}_{i}}$ operators [as shown in Fig. 6(b)], the number of fractons $F_{3, \mathbf{r}_{0}}$ at $\mathbf{r}_{0}$ is given by

$$
F_{3, \mathbf{r}_{0}}=\sum_{i_{1} \cdots i_{8}} a_{i_{1} \cdots i_{8}} \prod_{j} s_{j}^{i_{j}} .
$$

Here $a_{i_{1} \cdots i_{8}}$ are coefficients whose values can be found in Ref. [41]. The average number of fractons is then obtained that $\bar{F}_{3, \mathbf{r}_{0}}=29 / 32$, and the growth of $\mathcal{H}_{\mathrm{eff}, \mathrm{gg}^{\prime}}^{(N+\zeta)}$ for infinite $r$ is $256 v / 29 w$ as $r$ increases by one. Thus the upper critical point reads $v_{c 3}^{(u)}=29 w / 256$. An external term of strength $v>v_{c 2, c 3}^{u}$ drives the FTO into a trivial phase.

\section{B. Lower limits}

There remains an interesting issue on the lower limits of the critical point of the phase transition when the extra term can create higher dimensional (2D or 3D) fractons. The lower limit lies in identifying the growth of the leading-order contribution, whose exact solution is currently not available. However, we provide below a reasonable conjecture of the lower limits.

Conjecture: The critical points satisfy $v_{c 2} \geqslant w / 16$ and $v_{c 3}$ satisfies $v_{c 3} \geqslant w / 64$ when the extra term can create $2 \mathrm{D}$ and $3 \mathrm{D}$ fractons, respectively. The lower limits can be approached by estimating the maximum value of the transition matrix element between the ground states. The conjecture is based on the observation that for the $2 \mathrm{D}$ fractons, the number of configurations connecting two ground states in the same order perturbation is more than the case for $1 \mathrm{D}$ fractons due to the higher dimension in the operations. This is already seen in the exact study of the upper limits. The maximum number of the configurations could be the square of that for the case with 1D fractons, namely, the square of the Calatan number as shown in the case with lineons. In this case, the lower limit of the critical point is given by $v_{c 2}^{(l)}=w / 4^{2}=w / 16$. In a similar way, we conjecture that the lower limit for the case of 3D fractons is given by $v_{c 2}^{(l)}=w / 64$. The conjecture is clearly supported by numerical results, as given in the supplemental material [41]. To analytically confirm the lower limits and further the exact result of the critical point of phase transition in the generic case deserves future great efforts.

\section{CONCLUSION AND DISCUSSION}

We have presented an analytic theory of the quantum phase transition in FTO induced by external terms which create fracton excitations. We developed an effective Hamiltonian approach that is model independent to investigate the critical point of phase transition, which can be characterized by the breaking down of GSD. This approach can be mapped to the random-walk problem, allowing to study the critical point of quantum phase transition induced by fractons of different dimensions. For the lineonic external term, we provide a universal result of the critical point, given by $v_{c 1}=w / 4$ if $w_{i} \geqslant w$ or $v_{c 1}^{\prime} \approx \frac{w}{4}-\frac{w-w_{i}}{4} \times 0.26$ for $w_{i}<w$, with $w$ being the energy of a pair of lineons and $w_{i}$ being the energy of two lineons meeting at the same point. This result can be directly applied to the X-cube model with transverse Zeeman field [24]. For the external terms creating 2D or 3D fractons, e.g., the transverse field in the HHB model [25], we show an upper limit and conjectured a reasonable lower limit with numerical verification for the critical phase transition point. Beyond the upper limit (below the lower limit), the FTO breaks down (survives) in general. It should be noted that the quantum phase transition may also be characterized by level crossing between excited states and ground states, which render a firstorder transition. To develop analytic theory for such first-order transition is yet a challenging issue. Nevertheless, the analytic theory presented here shows insights into the understanding of the quantum phase transition in FTOs and opens the way to predict the full phase diagram of FTOs, which may also facilitate the physical realization of such exotic orders.

\section{ACKNOWLEDGMENTS}

This work was supported by National Natural Science Foundation of China (Grant No. 11825401), the Open Project of Shenzhen Institute of Quantum Science and Engineering (Grant No. SIQSE202003), and the Strategic Priority Research Program of Chinese Academy of Science (Grant No. XDB28000000) 


\section{APPENDIX: NTH-ORDER DEGENERATE PERTURBATION THEORY}

In this Appendix, we present the rigorous calculation of arbitrary $n$th order degenerate perturbation on the many-body ground states, whose degeneracy is assumed to be unchanged up to the $n-1$ th order. Let $H=H_{0}+\bar{v} V$, where $\bar{v} \ll 1, H_{0}$ denotes the unperturbed Hamiltonian, and $V$ is the external perturbation of the system. Write down that $H_{0}=H_{0, g g}+$
$H_{0, \bar{g} \bar{g}}$ and the external term $V=V_{g g}+V_{\bar{g} \bar{g}}+V_{g \bar{g}}+V_{\bar{g} g}$. The subscripts $g g$ (or $\bar{g} \bar{g}$ ) means that the corresponding operator is projected onto the ground-state subspace (or excited state subspace). Similarly, the subscripts $g \bar{g}$ and $\bar{g} g$ mean that the operators connect the ground and excited state subspaces.

Denote by $P$ the projection operator onto the ground-state subspace whose energy is $E$. For the total Hamiltonian $H$, the secular equation is

$$
\begin{aligned}
0= & \operatorname{det}\left(H_{0}+\bar{v} V-E\right) \\
= & \operatorname{det}\left\{\left[H_{0, \bar{g} \bar{g}}+\bar{v}\left(V_{\bar{g} \bar{g}}+V_{g \bar{g}}\right)-E(1-P)+P\right]\right. \\
& \left.\times\left[1-P+\bar{v}\left(1-\bar{v} V_{g \bar{g}}\right)\left(H_{0, \bar{g} \bar{g}}+\bar{v} V_{\bar{g} \bar{g}}-E(1-P)\right)_{e}^{-1} V_{\bar{g} g}+H_{0, g g}+\bar{v} V_{g g}-E P\right]\right\} \\
& \operatorname{det}\left[H_{0, g g}+\bar{v} V_{g g}-E-\bar{v}^{2} V_{g \bar{g}}\left(H_{0, \bar{g} \bar{g}}+\bar{v} V_{\bar{g} \bar{g}}-E\right)_{e}^{-1} V_{\bar{g} g}\right]_{g} \operatorname{det}\left(H_{0, \bar{g} \bar{g}}+\bar{v} V_{\bar{g} \bar{g}}-E\right)_{e} \\
& \operatorname{det}\left[H_{0, g g}+\bar{v} V_{g g}-E-\bar{v}^{2} V_{g \bar{g}}\left(\frac{1}{E H_{0, \bar{g} \bar{g}}}+\frac{1}{E-H_{0, \bar{g} \bar{g}}} \bar{v} V_{\bar{g} \bar{g}} \frac{1}{E H_{0, \bar{g} \bar{g}}}+\cdots\right) V_{\bar{g} g}\right]_{g} \\
& \times \operatorname{det}\left(H_{0, \bar{g} \bar{g}}+\bar{v} V_{\bar{g} \bar{g}}-E\right)_{e} .
\end{aligned}
$$

Then the effective Hamiltonian projected on the ground-state subspace is given by:

$$
\begin{aligned}
H_{\mathrm{eff}}= & H_{0, g g}+\bar{v} V_{g g}-\bar{v}^{2} V_{g \bar{g}}\left(\frac{1}{E-H_{0, \bar{g} \bar{g}}}\right. \\
& \left.+\frac{1}{E-H_{0, \bar{g} \bar{g}}} \bar{v} V_{\bar{g} \bar{g}} \frac{1}{E-H_{0, \bar{g} \bar{g}}}+\cdots\right) V_{\bar{g} g .}
\end{aligned}
$$

With the power series $E=\sum_{j} E_{j} \bar{v}^{j+1}$ we obtain that

$$
\begin{aligned}
\frac{1}{E-H_{0, \bar{g} \bar{g}}}= & \frac{1}{E_{0}-H_{0, \bar{g} \bar{g}}}-\bar{v} \frac{E_{1}}{\left(E_{0}-H_{0, \bar{g} \bar{g})^{2}}\right.} \\
& +\bar{v}^{2}\left(\frac{E_{1}^{2}}{\left(E_{0}-H_{0, \bar{g} \bar{g})^{3}}\right.}-\frac{E_{2}}{\left(E_{0}-H_{0, \bar{g} \bar{g})^{2}}\right.}\right)+\cdots \\
= & \frac{1}{E_{0}-H_{0, \bar{g} \bar{g}}}+\sum_{i=1}^{\infty} \sum_{s \in P_{i}} \frac{(-1)^{|s|} \bar{v}^{i} \prod_{j} E_{s_{j}}}{\left(E_{0}-H_{0, \bar{g} \bar{g}}\right)^{|s|+1}}
\end{aligned}
$$

where $E_{0}$ is the energy for the unperturbed ground state, $P_{i}$ is the set of integer partitions of the integer $i$, and the partition with different orders are counted different. For example, $1+2=2+1=3$ are two different partitions of $i=3$. In the summation $s_{j}$ is the $j$ th term in the integer partitions $s$ and $|s|$ is the number of integers partitioned. For example, if $s=3+1+5+4 \in P_{13}$, one has that $s_{3}=5, s_{4}=4$, and $|s|=4)$. For convenience, we further rewrite that $(E-$ $\left.H_{0, \bar{g} \bar{g}}\right)^{-1}=\sum_{i=0}^{\infty} \bar{v}^{i} Q^{(i+1)}$, with $Q^{(i)}$ in the expansion given by

$$
Q^{(i)}=-\sum_{s \in P_{i-1}} \frac{\prod_{j} E_{s_{j}}}{\left(H_{0, \bar{g} \bar{g}}-E_{0}\right)^{|s|+1}} .
$$

For example, the 4th-order term $Q^{(5)}$ of $\left(E-H_{0, \bar{g} \bar{g}}\right)^{-1}$ is $E_{1}^{4} / Q_{0}^{5}-3 E_{1}^{2} E_{2} / Q_{0}^{4}+E_{2}^{2} / Q_{0}^{3}+2 E_{3} E_{1} / Q_{0}^{3}-E_{4} / Q_{0}^{2}$, where we have defined $Q_{0} \equiv\left(Q^{(1)}\right)^{-1}=E_{0}-H_{0, \bar{g} \bar{g}}$. With these results the effective Hamiltonian projected onto the ground-state subspace can be further simplified in the following way

$$
\begin{aligned}
H_{\mathrm{eff}}= & H_{0, g g}+\bar{v} V_{g g}-\bar{v}^{2} V_{g \bar{g}} \frac{1}{Q_{0}} V_{\bar{g} g}-\bar{v}^{3} V_{g \bar{g}}\left(\frac{1}{Q_{0}} V_{\bar{g} \bar{g}} \frac{1}{Q_{0}}+Q^{(2)}\right) V_{\bar{g} g} \\
& -\bar{v}^{4} V_{g \bar{g}}\left(\frac{1}{Q_{0}} V_{\bar{g} \bar{g} \bar{g}} \frac{1}{Q_{0}} V_{\bar{g} \bar{g}} \frac{1}{Q_{0}}+Q^{(2)} V_{\bar{g} \bar{g}} \frac{1}{Q_{0}}+\frac{1}{Q_{0}} V_{\bar{g} \bar{g}} Q^{(2)}+Q^{(3)}\right) V_{\bar{g} g}-\cdots \\
= & H_{0, g g}+\bar{v} V_{g g}-\sum_{i=2}^{\infty} \bar{v}^{i} V_{g \bar{g}}\left(\sum_{\left(s_{1}, \cdots, s_{m}\right)=s \in P_{i-1}} Q^{\left(s_{1}\right)} V_{\bar{g} \bar{g} \bar{g}} Q^{\left(s_{2}\right)} V_{\bar{g} \bar{g}} \cdots V_{\bar{g} \bar{g}} Q^{\left(s_{m}\right)}\right) V_{\bar{g} g} .
\end{aligned}
$$


[1] A. P. Schnyder, S. Ryu, A. Furusaki, and A. W. W. Ludwig, Classification of topological insulators and superconductors in three spatial dimensions, Phys. Rev. B 78, 195125 (2008).

[2] A. Kitaev, Periodic table for topological insulators and superconductors, AIP Conf. Proc. 1134, 22 (2009).

[3] S. Ryu, A. P. Schnyder, A. Furusaki, and A. W. W. Ludwig, Topological insulators and superconductors: Tenfold way and dimensional hierarchy, New J. Phys. 12, 065010 (2010).

[4] X. Chen, Z.-C. Gu, Z.-X. Liu, and X.-G. Wen, Symmetryprotected topological orders in interacting bosonic systems, Science 338, 1604 (2012).

[5] X. G. Wen, Vacuum degeneracy of chiral spin states in compactified space, Phys. Rev. B 40, 7387 (1989).

[6] X. G. Wen, Topological order in rigid states, Int. J. Mod. Phys. B 04, 239 (1990).

[7] X. G. Wen and Q. Niu, Ground-state degeneracy of the fractional quantum Hall states in the presence of a random potential and on high-genus Riemann surfaces, Phys. Rev. B 41, 9377 (1990).

[8] A. Kitaev, Anyons in an exactly solved model and beyond, Ann. Phys. 321, 2 (2006).

[9] R. B. Laughlin, Anomalous Quantum Hall Effect: An Incompressible Quantum Fluid with Fractonally Charged Excitations, Phys. Rev. Lett. 50, 1395 (1983).

[10] S. M. Girvin, A. H. MacDonald, and P. M. Platzman, CollectiveExcitation Gap in the Fractional Quantum Hall Effect, Phys. Rev. Lett. 54, 581 (1985).

[11] G. Moore and N. Read, Nonabelions in the fractional quantum Hall effect, Nucl. Phys. B 360, 362 (1991).

[12] A. Kitaev, Fault-tolerant quantum computation by anyons, Ann. Phys. 303, 2 (2003).

[13] H. Bombin and M. A. Martin-Delgado, Topological Computation Without Braiding, Phys. Rev. Lett. 98, 160502 (2007).

[14] C. Nayak, S. H. Simon, A. Stern, M. Freedman, and S. D. Sarma, Non-Abelian anyons and topological quantum computation, Rev. Mod. Phys. 80, 1083 (2008).

[15] C. Nayak and F. Wilczek, 2n-quasihole states realize $2 n-1$ dimensional spinor braiding statistics in paired quantum Hall states, Nucl. Phys. B 479, 529 (1996).

[16] D. A. Ivanov, Non-Abelian Statistics of Half-Quantum Vortices in $p$-Wave Superconductors, Phys. Rev. Lett. 86, 268 (2001).

[17] S. Das Sarma, M. Freedman, and C. Nayak, Topologically Protected Qubits from a Possible Non-Abelian Fractional Quantum Hall State, Phys. Rev. Lett. 94, 166802 (2005).

[18] J. Alicea, Y. Oreg, G. Refael, F. von Oppen, and M. P. A. Fisher, Non-Abelian statistics and topological quantum information processing in 1D wire networks, Nat. Phys. 7, 412 (2011).

[19] X.-J. Liu, C. L. M. Wong, and K. T. Law, Non-Abelian Majorana Doublets in Time-Reversal-Invariant Topological Superconductors, Phys. Rev. X 4, 021018 (2014).

[20] J. Haah, Local stabilizer codes in three dimensions without string logical operators, Phys. Rev. A 83, 042330 (2011).

[21] S. Bravyi, B. Leemhuis, and B. M. Terhal, Topological order in an exactly solvable 3D spin model, Ann. Phys. 326, 839 (2011).
[22] C. Chamon, Quantum Glassiness in Strongly Correlated Clean Systems: An Example of Topological Overprotection, Phys. Rev. Lett. 94, 040402 (2005).

[23] B. Yoshida, Exotic topological order in fractal spin liquids, Phys. Rev. B 88, 125122 (2013).

[24] S. Vijay, J. Haah, and L. Fu, Fracton topological order, generalized lattice gauge theory, and duality, Phys. Rev. B 94, 235157 (2016).

[25] G. Halász, T. H. Hsieh, and L. Balents, Fracton Topological Phases from Strongly Coupled Spin Chains, Phys. Rev. Lett 119, 257202 (2017).

[26] H. Ma, E. Lake, X. Chen, and M. Hermele, Fracton topological order via coupled layers, Phys Rev. B 95, 245126 (2017).

[27] O. Petrova and N. Regnault, A simple anisotropic threedimensional quantum spin liquid with fracton topological order, Phys Rev. B 96, 224429 (2017).

[28] A. Prem, S.-J. Huang, H. Song, and M. Hermele, Cage-Net Fracton Models, Phys. Rev. X 9, 021010 (2019).

[29] D. J. Williamson, Fractal symmetries: Ungauging the cubic code, Phys. Rev. B 94, 155128 (2016).

[30] A. T. Schmitz, H. Ma, R. M. Nandkishore, and S. A Parameswaran, Recoverable information and emergent conservation laws in fracton stabilizer codes, Phys. Rev. B 97, 134426 (2018).

[31] H. He, Y. Zheng, B. Andrei Bernevig, and N. Regnault, Entanglement entropy from tensor network states for stabilizer codes, Phys. Rev. B 97, 125102 (2018).

[32] B. Shi and Y.-M. Lu, Deciphering the nonlocal entanglement entropy of fracton topological orders, Phys. Rev. B 97, 144106 (2018).

[33] S. Bravyi and J. Haah, Energy Landscape of 3D Spin Hamiltonians with Topological Order, Phys. Rev. Lett 107, 150504 (2011).

[34] S. Bravyi and J. Haah, Quantum Self-Correction in the 3D Cubic Code Model, Phys. Rev. Lett. 111, 200501 (2013).

[35] M. Prekto, Emergent gravity of fractons: Mach's principle revisited, Phys. Rev. D 96, 024051 (2017).

[36] A. Prem, J. Haah, and R. Nandkishore, Glassy quantum dynamics in translation invariant fracton models, Phys. Rev. B 95, 155133 (2017).

[37] M. Prekto, Higher-spin Witten effect and two-dimensional fracton phases, Phys. Rev. B 96, 125151 (2017).

[38] K. Slagle and Y. B. Kim, Quantum field theory of X-cube fracton topological order and robust degeneracy from geometry, Phys. Rev. B 96, 195139 (2017).

[39] W. Shirley, K. Slagle, Z. Wang, and X. Chen, Fracton Models on General Three-Dimensional Manifolds, Phys. Rev. X 8 , 031051 (2018).

[40] K. Slagle, D. Aasen, and D. Williamson, Foliated field theory and string-membrane-net condensation picture of fracton order, SciPost. Phys. 6, 043 (2019).

[41] See Supplemental Material at http://link.aps.org/supplemental/ 10.1103/PhysRevResearch.3.043114 for the details of the proof.

[42] M. Mühlhauser, M. R. Walther, D. A. Reiss, and K. P. Schmidt, Quantum robustness of fracton phases, Phys. Rev. B 101, 054426 (2020). 